\title{
Study On The Formation Mechanism of Cutting Surface of Carbon Fiber Reinforced Composites
}

Fei Su ( $\nabla$ sfeihe@163.com )

Hunan University of Science and Technology

Chunjie Li

Hunan University of Science and Technology

\section{Guojun Dong}

Harbin Institute of Technology

Lei Zheng

Yancheng Institute of Technology

\section{Bing Chen}

Hunan University of Science and Technology

\section{Research Article}

Keywords: Carbon fiber-reinforced plastic (CFRP), cutting surface, cutting mechanism, FEM modeling

Posted Date: November 17th, 2021

DOI: https://doi.org/10.21203/rs.3.rs-1076655/v1

License: (c) (i) This work is licensed under a Creative Commons Attribution 4.0 International License. Read Full License 


\title{
Study on the formation mechanism of cutting surface of carbon fiber reinforced composites
}

\author{
Fei Su${ }^{1 *}$, Chunjie $\operatorname{Li}^{1}$, Guojun Dong ${ }^{2 *}$, Lei Zheng ${ }^{3}$, Bing Chen ${ }^{1}$ \\ ${ }^{1}$ Hunan Provincial Key Laboratory of High Efficiency and Precision Machining of Difficult-to-Cut \\ Material, Hunan University of Science and Technology, Hunan Xiangtan 411201 \\ ${ }^{2}$ School of Mechatronics Engineering, Harbin Institute of Technology, Heilongjiang Harbin 150001 \\ ${ }^{3}$ School of Mechanical Engineering, Yancheng Institute of Technology, Jiangsu Yancheng 224051
}

Corresponding author: Tel: +86-0731-58291080; Fax: +86-0731-58291080; Email: sfeihe@163.com

Co-corresponding author: Tel: +86-0451-86416157; Fax: +86-0451-86416157;

Email: dongguojun@ @it.edu.cn

"This is an original paper which has neither previously, nor simultaneously, in whole or in part been submitted anywhere else." 


\title{
Study on the formation mechanism of cutting surface of carbon fiber reinforced composites
}

\begin{abstract}
Carbon fiber-reinforced plastic (CFRP) is used widely in aerospace. The cutting mechanism of CFRP is markedly different from that of metals due to anisotropic and non-homogeneous material structure. The cutting mechanisms are highly dependent on the fiber orientation. The quality of the machined surface can be affected by the fiber fracture models. In this paper, based on the elastic foundation beam theory and the Hertzian contact theory, the cutting mechanics are established. And the cutting model is simulated by the three-dimensional micro-scale numerical model. Then, the continuous varying cutting mechanism and the sub-damage are deeply studied in detail by combining the cutting mechanics model and the simulation model. The results indicate that the fiber orientation $\theta=80^{\circ}$ and $\theta=150^{\circ}$ is the transition critical point of the fracture form. When $\theta=0^{\circ}$, the fiber failure mode is buckling-dominated. When $0^{\circ}<\theta<80^{\circ}$ and $150^{\circ}<\theta<180^{\circ}$, the fiber failure mode is dominated by contact fracture. When $80^{\circ}<\theta<150^{\circ}$, the fiber failure mode is bending-dominated. The cutting mechanics model and finite element model can effectively reflect the evolution law of CFRP machined surface.
\end{abstract}

Keywords: Carbon fiber-reinforced plastic (CFRP); cutting surface; cutting mechanism; FEM modeling 


\section{Introduction}

Typically, carbon fiber-reinforced plastic (CFRP) is employed as the metals substitutions in lots of weight-critical components in aerospace, owing to the good properties, such as high specific strength, high specific stiffness and high modulus [1-5]. In reality, subsequent machining operations, such as milling, drilling, are necessary to be required for assembling [6]. However, some serious defects surrounding the cutting circumferences and inside the cutting wall are liable to produce, for example, delamination, cracking, fiber pull-out, etc. In general, the quality of the machined surface directly affects the machining quality of the component.

In general, the machinability of CFRP is markedly different from that of metals due to anisotropic and non-homogeneous material structure. The cutting process of CFRP was investigated in numerous existing studies. Wolfgang et al. [7] investigated the occurrence and propagation of milling defects. They found that the occurrence and propagation of milling defects were depend by the tool axis inclination and the fiber orientation. Seyedbehzad et al. [8] established a milling numerical model and pointed out that the extension of machining damage significantly depends on the fiber orientation. Mei et al. [9] carried out a series of milling experiments and established a milling force model for CFRP, according to the cutting conditions in different fiber orientation. Noritaka et al. [10] experimentally studied the internal defects generated on machined milled CFRP. They confirmed that the milling defects were generated based on the direction of fiber. Wang et al. [11] revealed the evolution laws of the fiber-matrix interface crack. They demonstrated that there were four evolution types. Noteworthy, the evolution type and depth were mainly determined by the fiber orientation.

All these exiting literatures attempted to explore the cutting mechanism by conducting tests, establishing theoretical cutting models and running simulation. Numerous studies confirmed that the 
chip formation, machined surface morphologies and fiber failure modes are highly dependent on the fiber orientation. Hu et al. [12] investigated the grinding performance of CFRP. The machined surface roughness was analyzed. Interestingly, they claimed that the surface roughness was affected by the fiber orientation and the worst surface roughness occurred at $\theta=120^{\circ}-150^{\circ}$. Additionally, the fiber fracture direction was mostly perpendicular to the fibers. Wang et al. [13] investigated the occurrence and formation mechanism of the surface cavity defects as $\theta$ ranging from $0^{\circ}$ to $180^{\circ}$. They suggested that the cavities were mainly caused by bending-induced and shear-induced fiber fracture, and the cavities could be avoided by employing "inclination milling". Niu et al. [14] proposed a micromechanical model. The fiber and matrix under different supporting conditions were discussed. Lukas et al. [15] established an analytical force model by considering the chip formation and the fiber failure mechanisms. The fiber failure and the tool-fiber contact situation were analyzed in their researches. Robert et al. [16] conducted the fiber fracture behavior and divided the fiber fracture model into four independent sub-models. They point out that the micro-bucking was the mainly fiber fracture model when the fiber orientation $\theta=0^{\circ} / 180^{\circ}$, the initial crack occurred at the contact point when $15^{\circ} \leqslant \theta \leqslant 75^{\circ}$, the fiber was broken by bending deformation when $\theta=90^{\circ}$, the fiber was "peeling" removed when $\theta$ was larger than $105^{\circ}$. Alessandro et al. [17-18] built a comprehensive three-dimensional micro-scale numerical model of UD-CFRP orthogonal cutting. The cutting processes with different fiber orientation $\left(\theta=0^{\circ}, 45^{\circ}, 90^{\circ}\right.$ and $\left.135^{\circ}\right)$ were carried out. Interestingly, they found that the damage area was orthogonal to the fiber orientation when $\theta=135^{\circ}$. Su et al. [19] established a three-dimensional finite element model to study the strain-rate-dependent mechanical properties of the resin and fiber. Then, the fiber fracture evolution processes of fiber and resin were characterized. Meng et al. [20] conducted a novel experiment and analyzed the machined surface roughness, sub-surface damage and burr 
formation. They indicated that the low machined surface roughness was observed when the fiber orientation was less than $80^{\circ}$ and the surface roughness increased rapidly as the fiber orientation went beyond $90^{\circ}$. The largest sub-damage was observed at around $135^{\circ}$.

All above exiting literatures contributed to explore the relationship between the fiber fracture and the fiber orientation. The cutting mechanism, the machined surface varying trend were described. However, few researches considered the fiber fracture situations by establishing the cutting mechanics model. Especially, the cutting mechanics model didn't combine the simulation model for analyzing the cutting mechanism with different fiber orientation in detail. In this paper, the cutting mechanics were established based on the elastic foundation beam theory and the Hertzian contact theory, the cutting model was simulated by the three-dimensional micro-scale numerical model. Then, the continuous varying cutting mechanism and the sub-damage, when the fiber orientation ranged from $0^{\circ}$ to $180^{\circ}$, were deeply studied in detail by combining the cutting mechanics model and the simulation model.

\section{Theoretical modeling of cutting surface formation}

\subsection{Cutting mechanics modeling of a single fiber}

Owing to the elastic deformation of the material during cutting, when the cutting depth $a_{\mathrm{p}} \leq \Delta=a_{\mathrm{p}}(1-K)$, the material only produces elastic deformation under the action of tool extrusion, but can't be removed [21]. In general, the machining-elasticity parameter $\mathrm{K}$ can be setted as 0.96 in here. Once the cutting depth $a_{\mathrm{p}}$ larger than this critical value, the material can be removed. The contact stress may be employed on the fiber when the tool nose is in contact with a fiber. Simultaneously, the bending stress may be produce. In carbon fiber-reinforced plastic (CFRP), the carbon fiber is the reinforcement. The mechanical properties of the fiber are quite different from those of the resin matrix. Obviously, the 
cutting fracture pattern of carbon fiber directly affects the cutting difficulty of CFRP. Then, the cutting mechanical model and the contact mechanics model of carbon fiber are established, as illustrated in Fig.

1.

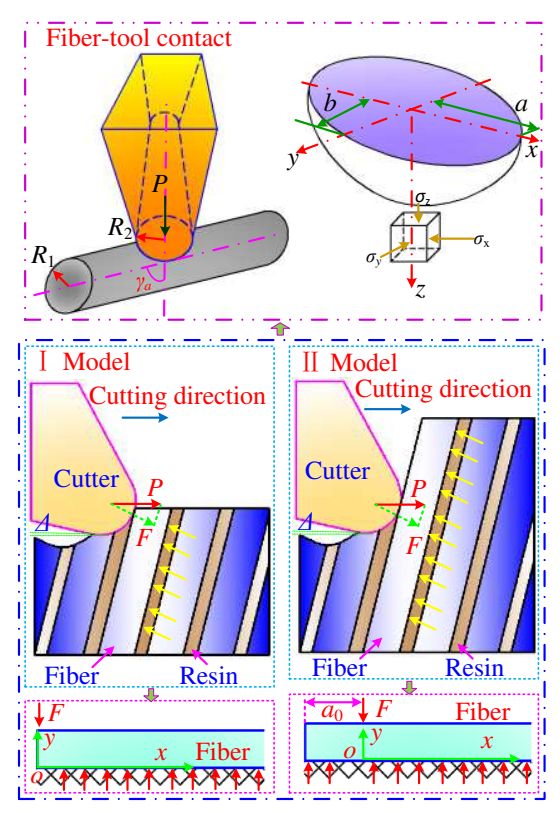

Fig.1 Cutting model of a single carbon fiber

(1) Tool-fiber contact model

According to the Hertz elasticity contact theory, the tool-fiber contact can be viewed as the contact between two circular cylinders. The geometry of contact region is an elliptical sphere. The Hertz solution yields the following expressions for the lengths of the long shaft $(a)$ and the short shaft $(b)$ of the ellipse (the major axis is oriented along the $x$-axis), as indicated in Eq. (1) [22].

$$
\left\{\begin{array}{l}
a=\eta_{a} \sqrt[3]{\frac{3 \eta P}{2 \Sigma \rho}} \\
b=\eta_{b} \sqrt[3]{\frac{3 \eta P}{2 \Sigma \rho}}
\end{array}\right.
$$

Moreover, the angle between the axis of the tool nose and the axis of the fiber is defined as $\gamma \mathrm{a}$.

Then, the principal relative curvatures (i.e. $\left.A_{0}, B_{0}\right)$ and the auxiliary function $F(\rho)$ can be listed out in Eq. (2) [22] 


$$
\left\{\begin{array}{l}
A_{0}+B_{0}=\frac{1}{2}\left(\frac{1}{R_{1 x}}+\frac{1}{R_{1 y}}+\frac{1}{R_{2 x}}+\frac{1}{R_{2 y}}\right)=\frac{1}{2} \Sigma \rho \\
A_{0}-B_{0}=\frac{1}{2} \sqrt{\left(\frac{1}{R_{1 x}}-\frac{1}{R_{1 y}}\right)^{2}+\left(\frac{1}{R_{2 x}}-\frac{1}{R_{2 y}}\right)^{2}+2\left(\frac{1}{R_{1 x}}-\frac{1}{R_{1 y}}\right)\left(\frac{1}{R_{2 x}}-\frac{1}{R_{2 y}}\right) \cos \left(2 \gamma_{a}\right)} \\
F(\rho)=\frac{A_{0}-B}{A_{0}+B_{0}}=1-\frac{2\left(1-e^{2}\right)}{e^{2}}\left(\frac{E(e)-K(e)}{E(e)}\right)
\end{array}\right.
$$

Where

$$
\left\{\begin{array}{l}
\eta_{a}=\sqrt[3]{\frac{2 E(e)}{\pi\left(1-e^{2}\right)}} \\
\eta_{b}=\eta_{a} \sqrt{\left(1-e^{2}\right)} \\
e=\sqrt{1-\left(\frac{b}{a}\right)^{2}}
\end{array}\right.
$$

Here, the maximum contact pressure $\left(p_{0}\right)$ is written as

$$
p_{0}=\frac{3 P}{2 \pi a b}
$$

$P$ is the total normal load acting on the ellipse; $e$ is the eccentricity of the ellipse; $K(e)$ and $E(e)$ are the complete elliptic integrals of the first and second kind; $R_{i x}$ and $R_{i y}(i=1$ or 2$)$ are the principal radius of the curvature of the ellipsoids at origin $\left(R_{i x}>R_{i y}\right)$.

Theoretically, it is very complex to calculate the internal stress field. Nevertheless, the maximum stress should occur on the $z$-axis at a point beneath the contact surface owing to the symmetry of the ellipsoid, and the shear stresses $\tau_{\mathrm{xy}}, \tau_{\mathrm{xz}}, \tau_{\mathrm{zy}}$ along the $z$-axis are all zero. To determine the fracture of the fiber during cutting and simplify the calculation, the maximum stress field can be determined by the following Eq. (5) [22].

$$
\left\{\begin{array}{l}
\sigma_{\mathrm{xs}}=-p_{0}\left[0.505+0.255(b / a)^{0.6609}\right] \\
\sigma_{\mathrm{ys}}=-p_{0}\left[1.01-0.25(b / a)^{0.5797}\right] \\
\sigma_{\mathrm{zs}}=-p_{0}
\end{array}\right.
$$

Thus, according to the 4th strength theory, the questions of mechanical failure of the fiber can be approached by calculating the von Mises yield parameter $\left(\sigma_{\mathrm{eq}}\right)$, as determined by Eq. (6). Based on the fracture criterion of maximum tensile stress, the fracture of the fiber occurs when $\sigma_{\mathrm{eq}}$ in the fiber 
exceeds the fiber tensile strength [21].

$$
\begin{aligned}
& \sigma_{e q}=\sqrt{\frac{1}{2}\left[\left(\sigma_{x}-\sigma_{y}\right)^{2}+\left(\sigma_{x}-\sigma_{z}\right)^{2}+\left(\sigma_{y}-\sigma_{z}\right)^{2}\right]} \\
& =p_{0} \sqrt{0.25-0.379(b / a)^{0.62}+0.192(b / a)^{1.24}}
\end{aligned}
$$

To resolve the stresses, first the auxiliary function $F(\rho)$ needs to be obtained according to the principal radius of the curvature of the ellipsoids. As in Yang et al. [23], the eccentricity of the ellipse $(e)$ can be estimated straightforwardly by the Eq. (7).

$$
\ln \left(\frac{1}{e}\right) \approx \begin{cases}0.4965 \ln \left[\frac{1}{F(\rho)}\right]-0.435 & (F(\rho) \leq 0.065) \\ 0.224\left[\ln \left(\frac{1}{F(\rho)}\right)\right]^{1.407} & (F(\rho)>0.065)\end{cases}
$$

(2) Cutting mechanical model

According to the actual cutting depth and cutting process, the cutting mechanical model can be simplified to two cases, viz., I model and II model, as depicted in Fig. 1.

In I model, the contact point is at the end of the fiber when the cutting depth is very small or after the fiber breaks. Then, according to the elastic foundation beam theory, the deflection $w(x)$ can be written as [24]

$$
w(x)=\frac{2 F \lambda}{k b} e^{-\lambda x} \cos (\lambda x)
$$

Subsequently, the bending load $F$ can be obtained by the deflection, as following

$$
F(x=0)=\frac{k b w}{2 \lambda e^{-\lambda x} \cos (\lambda x)}
$$

According to the mechanics of materials, the maximum internal stress $\sigma$ induced by the bending deformation can be determined by Eq. (10).

$$
\sigma=\frac{M_{\max } r_{\mathrm{f}}}{I_{\mathrm{f}}}
$$

Where

$$
M=\frac{4 E_{\mathrm{f}} I_{\mathrm{f}} F \lambda^{3} e^{-\lambda x} \sin (\lambda x)}{k b}
$$

When $\frac{d M}{d x}=0$, i.e., $w^{\prime \prime \prime}(x)=0$, the bending moment $M$ reaches the maximum. At this moment, the 
distance between the maximum bending point and the pressure point (the end of the fiber) can be calculated as

$$
L_{1}=\frac{\pi}{4 \lambda}
$$

Then, the maximum bending moment can be derived as Eq. (13). And the maximum compressive or tensile stress can be obtained as Eq. (14).

$$
\begin{gathered}
M_{\text {max }}=M(L)=\frac{4 E_{\mathrm{f}} I_{\mathrm{f}} F \lambda^{3} e^{-\frac{\pi}{4}} \sin \left(\frac{\pi}{4}\right)}{k b}=\frac{2 E_{\mathrm{f}} I_{\mathrm{f}} F \lambda^{3} e^{-\frac{\pi}{4}} \sqrt{2}}{k b} \\
\sigma_{1}=\frac{2 E_{\mathrm{f}} I_{\mathrm{f}} F \lambda^{3} e^{-\frac{\pi}{4}} \sqrt{2} r_{f}}{k b}
\end{gathered}
$$

Where

$$
\left\{\begin{array}{l}
\lambda=\sqrt[4]{\frac{k b}{4 E_{\mathrm{f}} I_{\mathrm{f}}}} \\
k=1.23\left[\frac{E_{m} d^{4}}{C E_{\mathrm{f}} I_{\mathrm{f}}\left(1-v_{m}^{2}\right)}\right]^{0.11} \frac{E_{m}}{C\left(1-v_{m}^{2}\right)} \\
I_{\mathrm{f}}=\frac{\pi\left(2 r_{\mathrm{f}}\right)^{4}}{64}
\end{array}\right.
$$

Where, $C$ is a coefficient varying from 1 for uniform pressure distribution to 1.13 for uniform deflection, here, $C=1.06$ [14]. $b, I_{\mathrm{f}}, E_{\mathrm{f}}, E_{\mathrm{m}}, v_{\mathrm{m}}, k, r_{\mathrm{f}}, d$ are the width of the beam, the moment inertia of the beam, the fiber elastic modulus, the matrix elastic modulus, the matrix Poisson's ratio, the modulus of the foundation, the fiber's radius, the fiber's diameter, respectively.

Similarly, in II model, the contact point is in the middle of the fiber when the cutting depth is large. The distance between the pressure point and the fiber end is assumed as $a_{0}$. The deflection of the fiber can be expressed as [24]

$$
\begin{aligned}
& w(x)=\frac{2 F \lambda e^{-\lambda|x|}}{k b}(\cos (\lambda|x|)+\sin (\lambda|x|)) \\
& +\frac{F \lambda}{k b}\left(\begin{array}{l}
e^{-\lambda a_{0}} \cos \left(\lambda a_{0}\right) e^{-\lambda\left(x+a_{0}\right)} \cos \left(\lambda\left(x+a_{0}\right)\right) \\
+\frac{1}{2} e^{-\lambda a_{0}}\left(\cos \left(\lambda a_{0}\right)-\sin \left(\lambda a_{0}\right)\right) e^{-\lambda\left(x+a_{0}\right)} \cos \left(\lambda\left(x+a_{0}\right)\right) \\
-\frac{1}{2} e^{-\lambda a_{0}}\left(\cos \left(\lambda a_{0}\right)-\sin \left(\lambda a_{0}\right)\right) e^{-\lambda\left(x+a_{0}\right)} \sin \left(\lambda\left(x+a_{0}\right)\right)
\end{array}\right)
\end{aligned}
$$


And the bending load on the pressure point can be derived as Eq. (17).

$$
F=\frac{k b w(x=0)}{2 \lambda e^{-\lambda|x|}(\cos (\lambda|x|)+\sin (\lambda|x|))+\lambda\left(\begin{array}{l}
e^{-\lambda a_{0}} \cos \left(\lambda a_{0}\right) e^{-\lambda\left(x+a_{0}\right)} \cos \left(\lambda\left(x+a_{0}\right)\right) \\
+\frac{1}{2} e^{-\lambda a_{0}}\left(\cos \left(\lambda a_{0}\right)-\sin \left(\lambda a_{0}\right)\right) e^{-\lambda\left(x+a_{0}\right)} \cos \left(\lambda\left(x+a_{0}\right)\right) \\
-\frac{1}{2} e^{-\lambda a_{0}}\left(\cos \left(\lambda a_{0}\right)-\sin \left(\lambda a_{0}\right)\right) e^{-\lambda\left(x+a_{0}\right)} \sin \left(\lambda\left(x+a_{0}\right)\right)
\end{array}\right)}
$$

Here, the breaking states of the fibers under the tool flank surface are taken into primary consideration, namely, $x>0$. Therefore, the bending moment $M$ can be written as

$$
M(x)=\frac{1}{k b}\left(\begin{array}{l}
E_{f} I_{f} F \lambda^{3}\left(3 e^{-\lambda\left(2 a_{0}+x\right)} \cos \left(\lambda a_{0}\right) \sin \left(\lambda\left(x+a_{0}\right)\right)\right. \\
+e^{-\lambda\left(2 a_{0}+x\right)} \cos \left(\lambda a_{0}\right) \cos \left(\lambda\left(x+a_{0}\right)\right) \\
-e^{-\lambda\left(2 a_{0}+x\right)} \sin \left(\lambda a_{0}\right) \sin \left(\lambda\left(x+a_{0}\right)\right) \\
-e^{-\lambda\left(2 a_{0}+x\right)} \sin \left(\lambda a_{0}\right) \cos \left(\lambda\left(x+a_{0}\right)\right) \\
\left.-4 e^{-\lambda x} \cos (\lambda x)+4 e^{-\lambda x} \sin (\lambda x)\right)
\end{array}\right)
$$

Similarly, when $\frac{d M}{d x}=0$, i.e., ${ }^{\prime \prime \prime \prime}(x)=0$, the bending moment $M$ reaches the maximum. The distance between the maximum bending point and the pressure point can be calculated as Eq. (19).

$$
L_{2}=\frac{1}{\lambda} \operatorname{atan}\left(\frac{4+e^{-2 \lambda a_{0}}-2 e^{-2 \lambda a_{0}} \cos \left(\lambda a_{0}\right) \sin \left(\lambda a_{0}\right)}{2 e^{-2 \lambda a_{0}}\left(\cos \left(e^{-2 \lambda a_{0}}\right)\right)^{2}}\right)
$$

Hence, the maximum bending moment and the maximum compressive or tensile stress can be derived as Eq. (20).

$$
\left\{\begin{array}{c}
M_{\max }=\frac{1}{k b}\left(\begin{array}{l}
E_{f} I_{f} F \lambda^{3}\left(3 e^{-\lambda\left(2 a_{0}+L_{2}\right)} \cos \left(\lambda a_{0}\right) \sin \left(\lambda\left(L_{2}+a_{0}\right)\right)\right. \\
+e^{-\lambda\left(2 a_{0}+L_{2}\right)} \cos \left(\lambda a_{0}\right) \cos \left(\lambda\left(L_{2}+a_{0}\right)\right) \\
-e^{-\lambda\left(2 a_{0}+L_{2}\right)} \sin \left(\lambda a_{0}\right) \sin \left(\lambda\left(L_{2}+a_{0}\right)\right) \\
-e^{-\lambda\left(2 a_{0}+L_{2}\right)} \sin \left(\lambda a_{0}\right) \cos \left(\lambda\left(L_{2}+a_{0}\right)\right) \\
\left.-4 e^{-\lambda L_{2}} \cos \left(\lambda L_{2}\right)+4 e^{-\lambda L_{2}} \sin \left(\lambda L_{2}\right)\right)
\end{array}\right) \\
\sigma_{2}=\frac{M_{\text {max }} \eta_{\max }}{I_{f}}=\frac{r_{f}}{k b I_{f}}\left(\begin{array}{l}
E_{f} I_{f} F \lambda^{3}\left(3 e^{-\lambda\left(2 a_{0}+L_{2}\right)} \cos \left(\lambda a_{0}\right) \sin \left(\lambda\left(L_{2}+a_{0}\right)\right)\right. \\
+e^{-\lambda\left(2 a_{0}+L_{2}\right)} \cos \left(\lambda a_{0}\right) \cos \left(\lambda\left(L_{2}+a_{0}\right)\right) \\
-e^{-\lambda\left(2 a_{0}+L_{2}\right)} \sin \left(\lambda a_{0}\right) \sin \left(\lambda\left(L_{2}+a_{0}\right)\right) \\
-e^{-\lambda\left(2 a_{0}+L_{2}\right)} \sin \left(\lambda a_{0}\right) \cos \left(\lambda\left(L_{2}+a_{0}\right)\right) \\
\left.-4 e^{-\lambda L_{2}} \cos \left(\lambda L_{2}\right)+4 e^{-\lambda L_{2}} \sin \left(\lambda L_{2}\right)\right)
\end{array}\right)
\end{array}\right.
$$

Additionally, the bending load $F$ perpendiculars to the fiber axial during the cutting of the fibers.

Therefore, the relationship between the contact load $P$ and the bending load $F$ can be mathematically 
expressed in function of $P=F \cos \left(\varphi^{\prime}\right)$. According to the angle $\theta$ between the fiber axial and the cutting direction, namely, the fiber direction, the cutting states can be divided into two cases, i.e., $0^{\circ}<\theta<90^{\circ}$ 和 $90^{\circ}<\theta<180^{\circ}$, as illustrated in Fig. 2. Here, the angle $\varphi^{\prime}$ between the cutting direction and the bending load direction can be determined by $\varphi$ and $\theta$, as expressed as Eq. (21). The symbol $\varphi$ is the initial angle between the cutting direction and the bending load direction, and the symbol $\delta$ is the bending angle, $\delta \approx w / L$.

$$
\varphi^{\prime}=\left\{\begin{array}{lc}
\frac{\pi}{2}-\theta+\delta=\varphi+\delta & 0^{\circ}<\theta<90^{\circ} \\
\theta-\frac{\pi}{2}-\delta=\varphi-\delta & 90^{\circ}<\theta<180^{\circ}
\end{array}\right.
$$

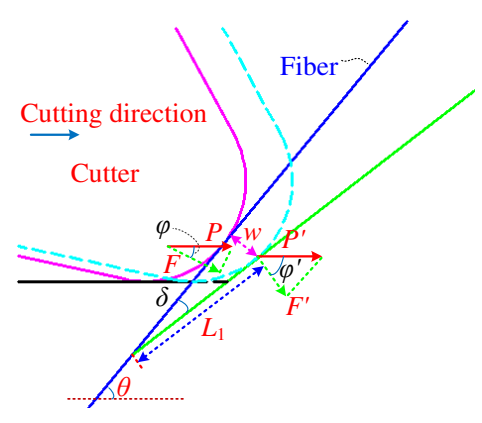

(a) When $0^{\circ}<\theta<90^{\circ}$

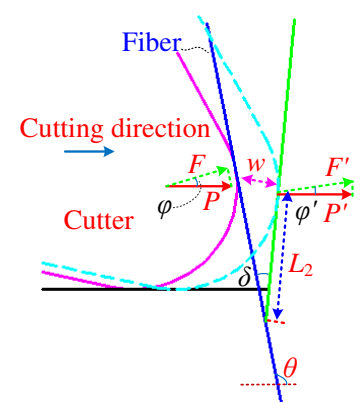

(b) When $90^{\circ}<\theta<180^{\circ}$

Fig.2 Cutting states of the fiber

\subsection{Mechanical modeling of cutting surface formation}

Essentially, the cutting states can be subdivided into 8 typical cases based on the changes of the fiber direction $\theta$ and the cutting depth $a_{\mathrm{p}}$, when $0^{\circ}<\theta<180^{\circ}$, as depicted in Fig. 3. Those typical cases may be transformed each other when the cutting depth $a_{\mathrm{p}}$ exceeds the critical cutting depth $a_{\mathrm{c}}$. Based on the geometrical relationship, obviously, the critical cutting depth $a_{\mathrm{c}}$ can be determined by the blunt radius $r_{\mathrm{e}}$ and the fiber direction $\theta$, namely, $a_{\mathrm{c}}=r_{\mathrm{e}}(1-\cos \theta)$, when $0^{\circ}<\theta \leq 90^{\circ}$. When $a_{\mathrm{p}} \leq a_{\mathrm{c}}$, the contact point is at the end of the fiber, the cutting mechanics model can be solved by I model. Otherwise, the cutting mechanics model can be solved by II model. Similarly, when $0^{\circ}<\theta<180^{\circ}$, the 
critical cutting depth $a_{\mathrm{c}}$ can be derived by the blunt radius $r_{\mathrm{e}}$ and the rake angle $\gamma_{0}$, namely, $a_{\mathrm{c}}=r_{\mathrm{e}}\left(1+\sin \gamma_{0}\right)$. When $90^{\circ}<\theta \leq 90^{\circ}+\gamma_{0}$, if $a_{\mathrm{p}} \leq a_{\mathrm{c}}$, the contact point is at the end of the fiber, the cutting mechanics model can be solved by I model, too. Otherwise, the cutting mechanics model can be solved by II model. But when $90^{\circ}+\gamma_{0}<\theta<180^{\circ}$, the contact point is always at the fiber end. Then, the cutting mechanics model can be only solved by I model. Generally, the fiber must break at the contact point or under the contact point for II model. Hereafter, the cutting mechanics model of II model must convert into I model.
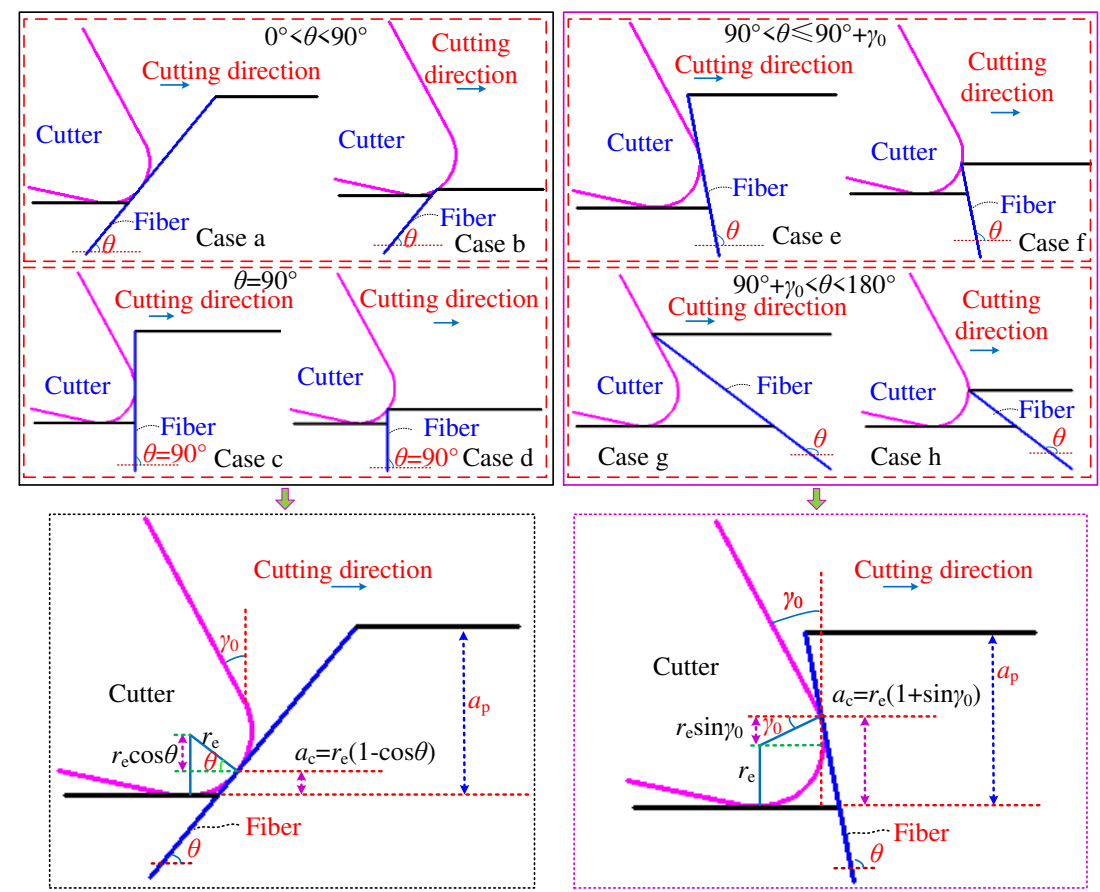

Fig.3 Typical refined cases

According to numerous studies, some cavities may be observed on the machined surface when $90^{\circ}<\theta<180^{\circ}$, because the breaking point is under the tool. And the overall fracture surface of the fibers basically perpendiculars to the fiber axial [12, 17-18]. Thus, the breaking depth of a fiber $\Delta h$ can be calculated as Eq. (22), according to the cutting geometry model, as presented in Fig. 4.

$$
\Delta h=j L \sin (\pi-\theta)-a_{p}
$$


Where, the symbol $j L$ can be expressed as

$$
j L=\left\{\begin{array}{l}
L_{2}+n L_{1} \quad a_{p}>a_{c} \\
n L_{1} \quad a_{p} \leq a_{c}
\end{array}\right.
$$

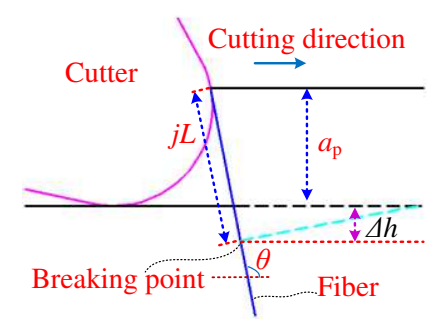

Fig.4 Cutting geometry model when $90^{\circ}<\theta<180^{\circ}$

\subsection{Cutting finite element modeling}

In order to analyze and compare the fibers cutting process, the micro-scale orthogonal cutting simulations are established.

A series of micro-scale orthogonal cutting 3D models with specified fiber orientations $\left(\theta=0^{\circ}, 45^{\circ}\right.$, $90^{\circ}, 135^{\circ}, 110^{\circ}, 160^{\circ}$, etc.) are established by the commercial software ABAQUS. In these models, all the tool, fibers and resin are meshed by C3D8R units. The user-defined 3D damage model of the fibers is implemented by VUMAT. In this model, the fibers are assumed to fail and removed, after the axial maximum principal stress meets the tensile strength or the minor principal stress is reached the compressive strength. The resin epoxy matrix is characterized as isotropic elastoplastic material. The shear failure criterion is used as the initial failure criterion of the resin. The cohesive element with zero thickness is usually applied to simulate interface behavior between different CFRP layers.

\section{Experimental verification}

To validate the theoretical model established in the previous sections and analyze the formation mechanisms of the machined surface, a series of milling tests were carried out on KVC1050M vertical machining center without coolant, by the end mill made of YG6X carbide without coating, with 4 flutes 
and a diameter $6 \mathrm{~mm}$. The milling parameters of $64 \sim 109 \mathrm{~m} / \mathrm{min}$ in cutting speed and 100 580 $\mathrm{mm} / \mathrm{min}$ in feed speed were used. The cutting depth was $3 \mathrm{~mm}$. All the experimental setups and the warp fibers were depicted in Fig.5. After tests, the microstructures of groove wall were observed and analyzed by scanning electron microscope (SEM).

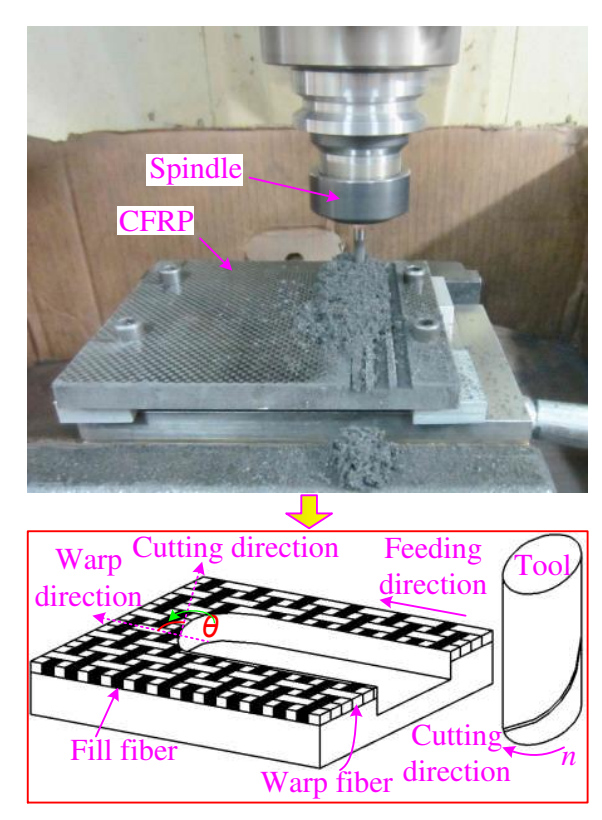

Fig. 5 Experimental setups and warp fiber

The plain woven carbon fiber reinforced plastics (CFRP) with about 10 15 mm thickness were employed in all these experiments. The fibre diameter was about $7 \sim 8 \mu \mathrm{m}$. The fiber volume content was about $60 \sim 65 \%$. Each ply had a thickness of $0.25 \mathrm{~mm}$. The material properties of CFRP and tool were listed in Table 1.

Table 1 Properties of CFRP and tool

\begin{tabular}{cc}
\hline Parameter & Value \\
\hline Fiber elastic modulus & $E_{1}=235 \mathrm{Gpa}, E_{2}=E_{3}=14 \mathrm{GPa}$ \\
$v_{12}=v_{13}=0.2, v_{23}=0.25$ \\
Fiber Poisson ratio & $G_{12}=G_{13}=28 \mathrm{Gpa}, G_{23}=5.5 \mathrm{GPa}$ \\
Fiber shear modulus & $X_{\mathrm{t}}=3.72 \mathrm{GPa}$ \\
Fiber tensile strength & $X_{\mathrm{c}}=3 \mathrm{GPa}$ \\
Fiber compressive strength & $E_{\mathrm{m}}=2.96 \mathrm{GPa}$ \\
Matrix elastic modulus & $\nu=0.4$ \\
Matrix Poisson ratio & $\sigma_{\mathrm{y} 0}=74.4 \mathrm{MPa}$ \\
Matrix yield strength &
\end{tabular}


Interface properties

Cutter rake angle

Cutter clearance angle

Cutter blunt round radius $t_{\mathrm{n}}^{0}=60 \mathrm{Mpa}, t_{\mathrm{s}}^{0}=t_{\mathrm{t}}^{0}=110 \mathrm{Mpa}$

$G_{\mathrm{n}}^{\mathrm{c}}=0.33\left(\mathrm{~N} / \mathrm{mm}^{2}\right), G_{\mathrm{c}}^{\mathrm{s}}=G_{\mathrm{t}}^{\mathrm{c}}=1.209\left(\mathrm{~N} / \mathrm{mm}^{2}\right)$

$K_{\mathrm{nn}}=4 \times 10^{6}\left(\mathrm{~N} / \mathrm{mm}^{2}\right), K_{\mathrm{ss}}=K_{\mathrm{tt}}=1 \times 10^{6}\left(\mathrm{~N} / \mathrm{mm}^{2}\right)$

$20^{\circ}$

$5^{\circ}$

$10 \mu \mathrm{m}$

\section{Results and discussion}

\subsection{Cutting process of a single fiber}

Combining the cutting mechanics model and the finite element model, the cutting process of the

fibers with specified fiber orientations $\left(\theta=0^{\circ}, 45^{\circ}, 90^{\circ}, 135^{\circ}\right.$, etc.) is analyzed, as presented in Fig. 6

(a) $\sim($ d)

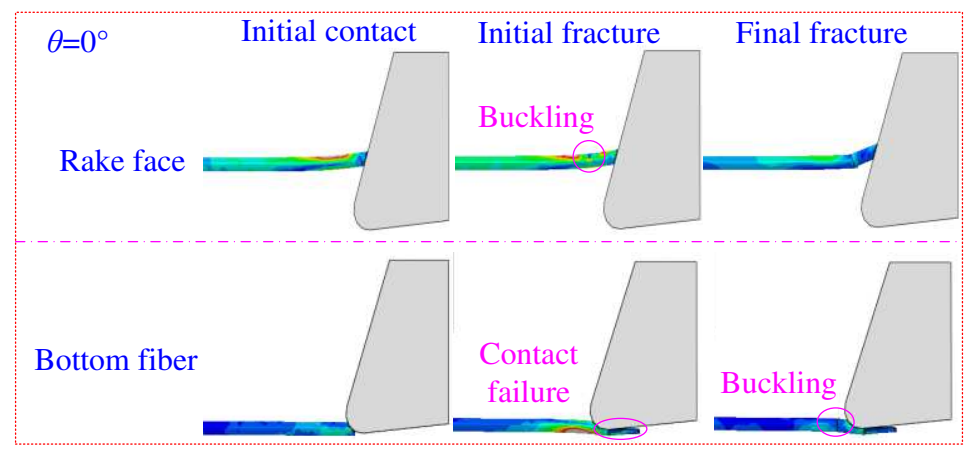

(a) Fiber cutting process when $\theta=0^{\circ} / 180^{\circ}$

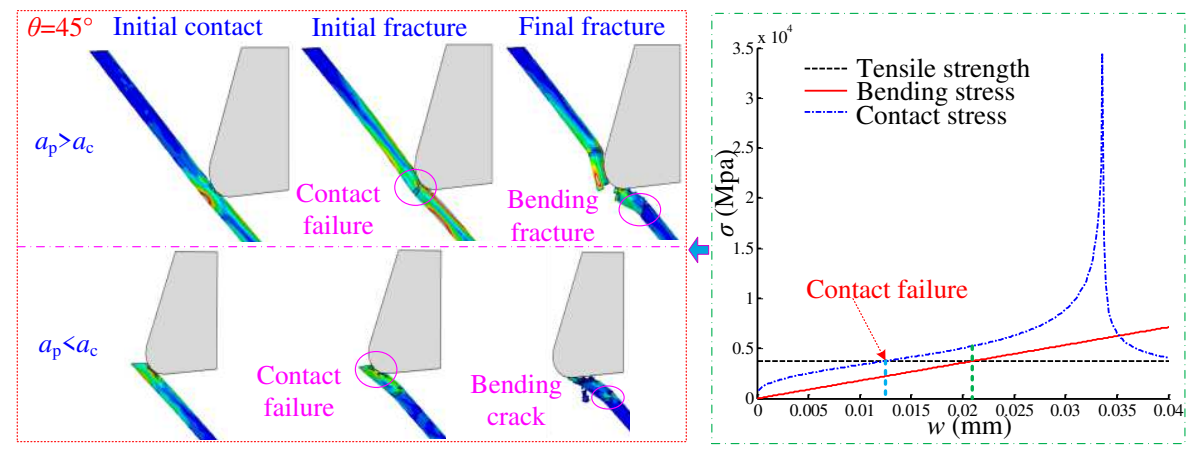

(b) Fiber cutting process when $\theta=45^{\circ}$ 

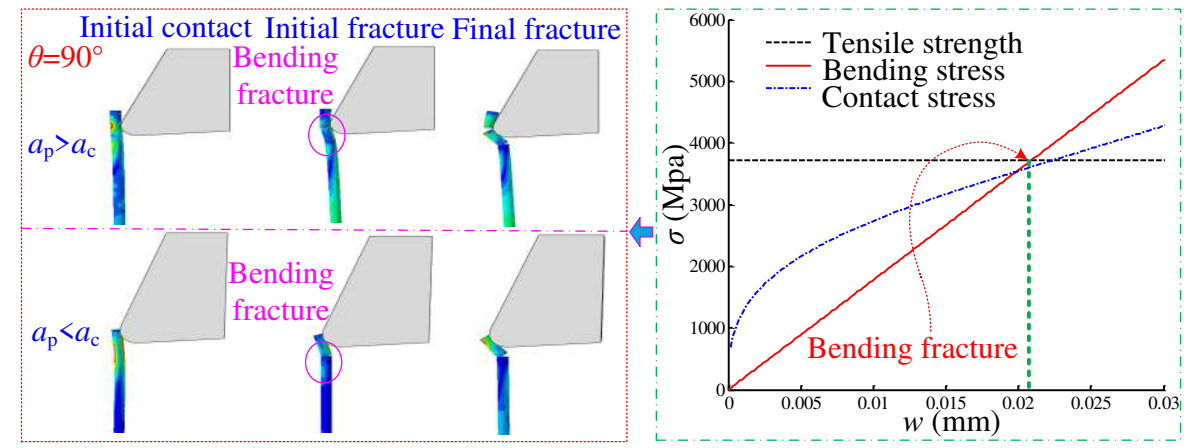

(c) Fiber cutting process when $\theta=90^{\circ}$
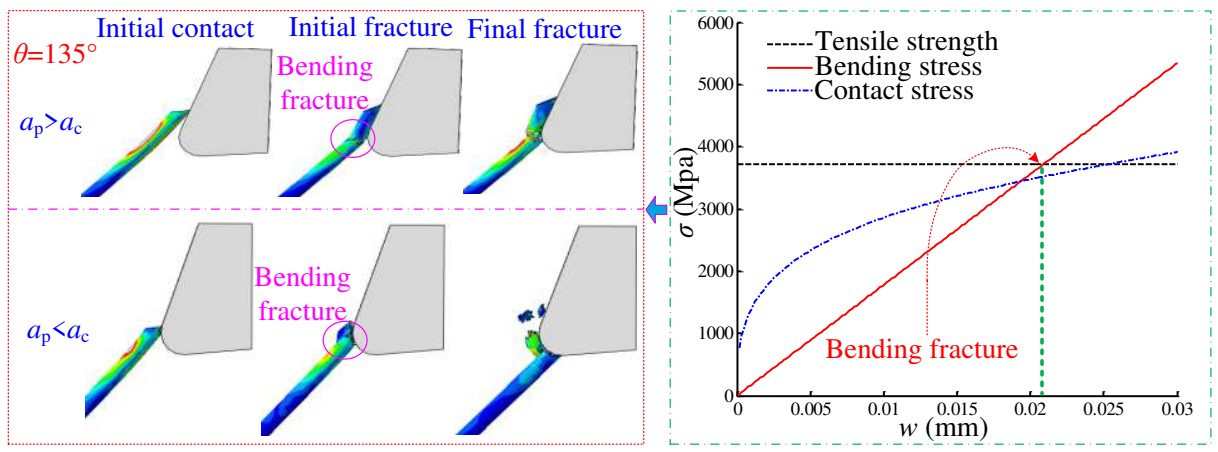

(d) Fiber cutting process when $\theta=135^{\circ}$

Fig.6 Fiber cutting processes with different fiber orientation

When $\theta=0^{\circ} / 180^{\circ}$, the load acted on the fiber end is basically parallel to the fiber axial. Thus, the buckling deformation occurs. Nevertheless, there are significant differences in fibers fracture situations because of the differences in the state of loads, when the cutter-fiber contact points are on the cutter rake face or at the bottom of the cutter, as depicted in Fig. 6 (a). When the cutter-fiber contact points are on the cutter rake face, the load acted on the fiber end is basically parallel to the fiber axial and the fiber buckling deformation occurs. Simultaneously, the fiber bends upward and the compression fracture cracks occur on the fiber surface, owing to the tilt of the cutter rake face. Then, the fiber buckling fracture failure is formed. When the cutter-fiber contact points are at the bottom of the cutter, while the fiber buckling deformation occurs, the fiber is squeezed back to the cutter flank surface due to the influence of the cutter blunt circular. As a result, the fibers at the cutter bottom is squeezed and 
broken in the form of compression fracture failure. Meanwhile, the fiber in front of the cutter is bent and buckled in the form of buckling fracture. Additionally, the fiber in the contact area is squeezed and broken in the form of contact fracture failure.

When $\theta=45^{\circ}$, two types of cutting conditions with $a_{\mathrm{p}}>a_{\mathrm{c}}$ and $a_{\mathrm{p}}<a_{\mathrm{c}}$ are contrastive investigated, as depicted in Fig. 6 (b). In Fig. 6 (b), when $\theta=45^{\circ}$, the initial fracture basically occurs at the cutter-fiber contact point. As the cutter continues to push, the fibers at the cutter bottom is bent. When $a_{\mathrm{p}}>a_{\mathrm{c}}$, the fibers at the cutter bottom can be bent and broken. But only cracks occur in these fibers at the cutter bottom when $a_{\mathrm{p}}<a_{\mathrm{c}}$. According to the cutting mechanics model, the von Mises yield stress $\left(\sigma_{\mathrm{eq}}\right)$ is much larger than the maximum bending stress when $\theta=45^{\circ}$. The von Mises yield stress $\left(\sigma_{\mathrm{eq}}\right)$ firstly exceeds the breaking strength. Therefore, the contact fracture failure has already happened before the bending fracture. Hence, the contact fracture is the main failure when $\theta=45^{\circ}$.

When $\theta=90^{\circ}$, two types of cutting conditions with $a_{\mathrm{p}}>a_{\mathrm{c}}$ and $a_{\mathrm{p}}<a_{\mathrm{c}}$ are also contrastive studied, as illustrated in Fig. 6 (c). When $a_{\mathrm{p}}>a_{\mathrm{c}}$, the fiber under the cutter is bent and broken. And the fiber contact fracture produces at the contact point. The bending fracture and the contact fracture occur almost at the same time. Nevertheless, the fiber contact stress at the contact point is less than the fiber bending stress at the cutter bottom when $a_{\mathrm{p}}<a_{\mathrm{c}}$. Then, there is no significant contact fracture at the contact point. The initial bending fracture of the fiber occurs under the cutter. Noteworthy, comparing with the von Mises yield stress $\left(\sigma_{\mathrm{eq}}\right)$, the maximum bending stress exceeds the breaking strength slightly earlier. But these two points beyond the breaking strength are close. Therefore, the bending fracture with contact fracture is the main initial failure. The fiber fracture ends left on the machined surface are mainly bending fracture ends.

Similarly, when $\theta=135^{\circ}$, two types of cutting conditions with $a_{\mathrm{p}}>a_{\mathrm{c}}$ and $a_{\mathrm{p}}<a_{\mathrm{c}}$ are researched, too. 
As shown in Fig. 6(d), the initial fracture all occurs at the cutter bottom. The fiber contact stress at the contact point is far less than the fiber bending stress at the cutter bottom. Thus, the significant bending fracture occurs. Based on the cutting mechanics model, comparing to the von Mises yield stress $\left(\sigma_{\mathrm{eq}}\right)$, the maximum bending stress exceeds the breaking strength obviously earlier, when $\theta=135^{\circ}$. And these two points beyond the breaking strength are far away. Then, the initial fracture of fiber is mainly bending fracture.

Based on the cutting mechanics model, the von Mises yield stress $\left(\sigma_{\mathrm{eq}}\right)$ and the maximum bending stress are investigated when $\theta=80^{\circ}$ and $\theta=150^{\circ}$, as presented in Fig. 7. When $\theta=80^{\circ}$ and $\theta=150^{\circ}$, the von Mises yield stress $\left(\sigma_{\mathrm{eq}}\right)$ and the maximum bending stress meets the breaking strength almost simultaneously. When the fiber orientation $\theta$ is larger than $80^{\circ}$, the fiber fracture may be changed from contact fracture to bending fracture. Conversely, when the fiber orientation $\theta$ is larger than $150^{\circ}$, the fiber fracture may be changed from bending fracture to contact fracture. Therefore, the fiber orientation $\theta=80^{\circ}$ and $\theta=150^{\circ}$ is the transition critical point of the fracture form. At the critical point, the contact fracture and the bending fracture will occur simultaneously. At this time, many comminuted fiber fractures will appear on the machined surface.
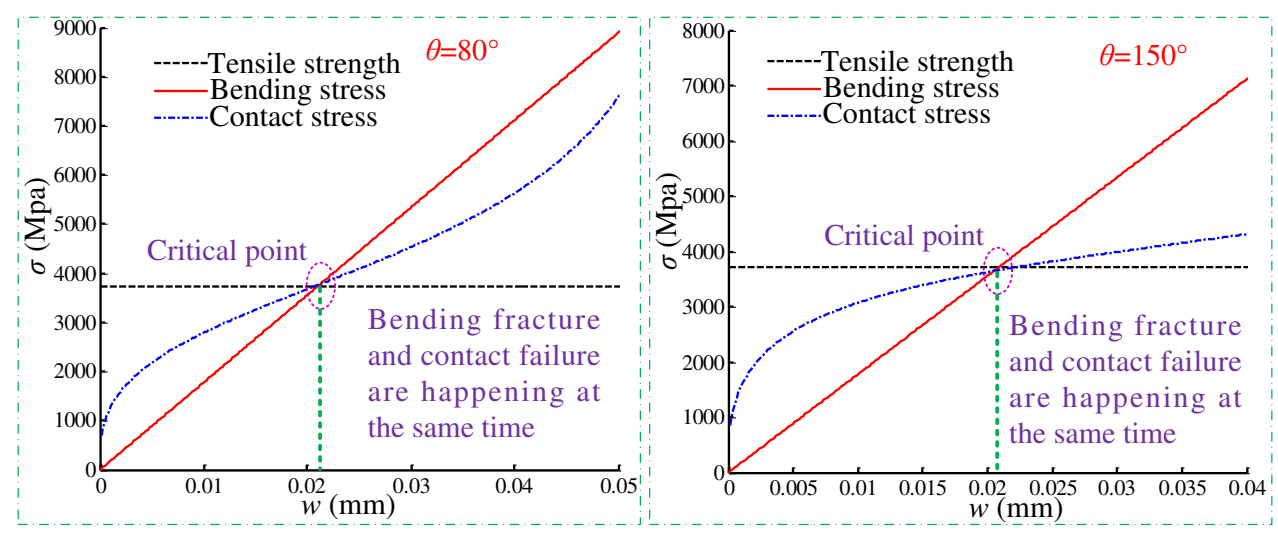

Fig.7 Contact and bending stress when $\theta=80^{\circ}$ and $\theta=150^{\circ}$

In summary, the fiber orientation $\theta=80^{\circ}$ and $\theta=150^{\circ}$ is the transition critical point of the fracture 
form. When $\theta=0^{\circ}$, the fiber failure is mainly buckling fracture and the breaking point of fiber is basically at the contact point. When $0^{\circ}<\theta<80^{\circ}$, the fiber failure is mainly contact fracture and the breaking point of fiber is basically at the contact point. When $80^{\circ}<\theta<150^{\circ}$, the fiber failure is mainly bending fracture and the fiber breaking point is under the cutter. Then, the "cavity" machined surface may be even formed. When $150^{\circ}<\theta<180^{\circ}$, the fiber failure is mainly contact fracture and the breaking point of fiber is basically at the contact point. Noteworthy, the fiber failure is not entirely dominated by contact fracture when $\theta \leq 90^{\circ}$. The fiber failure is not entirely dominated by bending fracture when $\theta>90^{\circ}$.

\subsection{Formation mechanism of cutting surface}

The morphologies of the machined surface within $80^{\circ}<\theta<150^{\circ}$ and $150^{\circ}<\theta<180^{\circ}$ are observed.

The morphologies of the machined surface when $\theta=110^{\circ}$ and $\theta=160^{\circ}$ are reported in Fig. 8 .

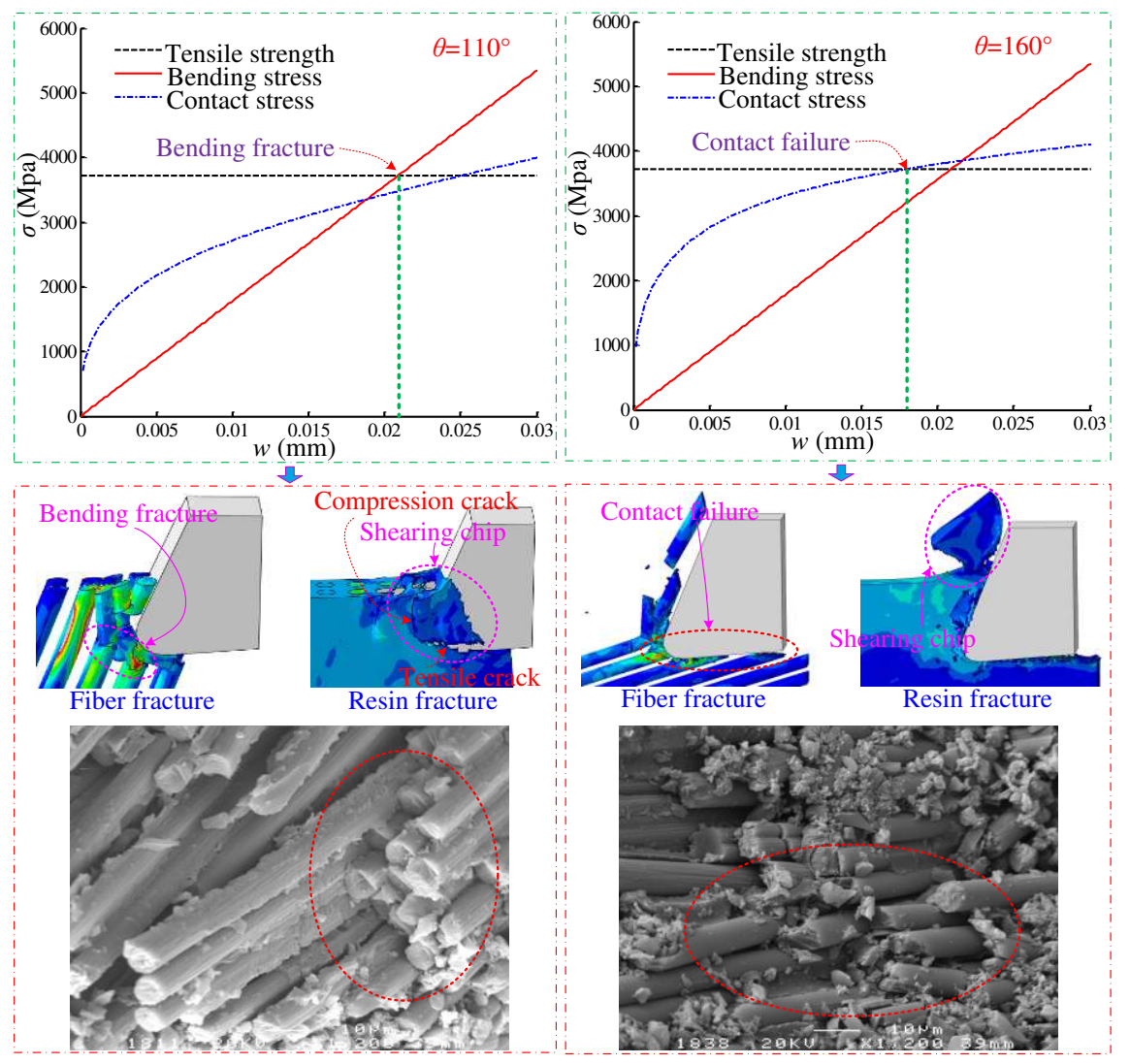

Fig. 8 Cutting process of CFRP when $\theta=110^{\circ}$ and $\theta=160^{\circ}$ 
When $\theta=110^{\circ}$, the maximum bending stress firstly meets the breaking strength. The point beyond the breaking strength of the von Mises yield stress $\left(\sigma_{\mathrm{eq}}\right)$ is far behind that of the maximum bending stress. Through the observation of the cutting process, the fiber breaking point is mainly at the cutter bottom, but not at the contact point. Obviously, the fiber failure is mainly the bending fracture. In addition, the resin matrix in the front of the cutter is squeezed and broken. The resin matrix at the bottom of the cutter is stretched and broken. The "cavities" machined surface is formed because the fracture point is under the cutter, owing to the bending fracture. The machined surface is uneven, but the fiber fracture ends are neat and clear.

Conversely, when $\theta=160^{\circ}$, the von Mises yield stress $\left(\sigma_{\mathrm{eq}}\right)$ firstly exceeds the breaking strength. The point beyond the breaking strength of the maximum bending stress is far behind that of the von Mises yield stress $\left(\sigma_{\mathrm{eq}}\right)$. Similarly, through the observation of the cutting process, obvious contact fragmentation occurs at the contact point and the morphology of fiber fracture is irregular. The resin matrix fracture occurs in the extrusion and tensile state, too. Therefore, the fiber failure is mainly contact fracture and the fiber fragmentation occurs. The fiber fracture ends are irregular, but the machined surface is smooth. The fiber fracture fragmentation can also be observed through the microscopic morphologies of the machined surface. Therefore, the results obtained from the fiber cutting mechanics model and the finite element cutting model are in good agreement with the actual machining.

The machined surface formation and morphology with specified fiber orientations $\left(\theta=0^{\circ}, 45^{\circ}, 90^{\circ}\right.$, $135^{\circ}$, etc.) are investigated, as displayed in Fig. $9(\mathrm{a}) \sim(\mathrm{d})$. The results can be found through the finite element cutting process and microscopic morphology observation, as following: 1) As revealed above, when $\theta=0^{\circ}$, the fiber is mainly broken by the buckling, accompanied by contact breakage. The chips 
form is mainly the crushed powder chips. The machined surface is smooth. During the milling, when the tool cut into and cut out the workpiece, the warp fiber directions are equal to $0^{\circ}$ and $180^{\circ}$ (i.e., $\theta=0^{\circ}$, $\theta=180^{\circ}$ ), respectively. The cutting models of these two fiber orientations are basically the same. The machined surface morphologies of these two fiber orientations are similar. The machined surfaces of these two fiber orientations are relatively flat. However, there are some differences in fiber fracture ends, when the tool cut into and cut out the workpiece (i.e., $\theta=0^{\circ}, \theta=180^{\circ}$ ). When the tool cut into the workpiece $\left(\theta=0^{\circ}\right)$, the fiber fracture ends are relatively neat. When the tool cut out the workpiece $\left(\theta=180^{\circ}\right)$, the fiber fracture ends are relatively sharp and irregular. 2) When $\theta=45^{\circ}$, a large number of contact broken fibers appear at the front of the cutter and some compressed broken fibers appear at the bottom of the cutter. The fiber fracture surface is neat and clear. The machined surface is relatively smooth. 3) When $\theta=90^{\circ}$, a large number of compressed broken fibers appear at the front of the cutter. The fibers at the bottom of the cutter are obviously bent and broken. The fracture fibers can't be completely removed due to the wraparound effect of the resin matrix. Then, the overall machined surface is relatively flat, but the fiber fracture ends are blurred because of the most of broken fibers. The roots of most fibers are basically broken. 4) When $\theta=135^{\circ}$, the fibers at the front of the cutter are basically bent and broken. The fibers below of the cutter are partly bent and broken. The machined surface isn't smooth. Even there are some "cavities" appear on the machined surface. But the fiber fracture ends are clear. Additionally, the contact fracture direction of the fiber is basically parallel to the cutting direction, and the machined surface is relatively flat. However, the bending fracture direction of the fiber is basically perpendicular to the fiber axial. The breaking point is usually below the machined surface and the machined surface is easy to form an uneven morphology.

Generally, when the warp fiber direction $\theta$ is near $0^{\circ}, 45^{\circ}, 90^{\circ}$ and $180^{\circ}$, the machined surface of 
the milling groove wall is relatively smooth. But the machined surface isn't smooth when the warp fiber direction $\theta$ is near $135^{\circ}$. Some "cavities" can be observed on the machined surface when $\theta$ is near $135^{\circ}$. When $\theta$ is near $0^{\circ}$ or $180^{\circ}$, the fiber and resin interface is broken and separated at first. Subsequently, the fibers in front of the cutter are broken by the extrusion and bending of the cutter. The overall machined surface is smooth and the fiber fracture ends are clear. When $\theta$ is near $45^{\circ}$, the machined surface is the smoothest. The fiber fracture ends are even. The machined surface is evenly covered by the resin. There are few "cavities" can be observed on the machined surface. But there are some broken fibers can be observed. When $\theta$ is near $90^{\circ}$, the machined surface is smooth and clean. The fiber fragmentations and the root fracture fibers can be obviously observed on the machined surface. The fiber and resin are all damaged to a certain extent. When $\theta$ is near $135^{\circ}$, the brittle fracture occurs in the fiber because of the compression and bending. Most of the fiber breaking point occurs at the maximum bending stress. Then, more "cavities" can be observed on the machined surface. Hence, the machined surface quality is poor. As can be seen from the above studies, when $0^{\circ} \leq \theta \leq 80^{\circ}$, the fiber fracture is mainly caused by the contact fracture failure. The machined surface is generally smooth. When $80^{\circ}<\theta \leq 150^{\circ}$, the fiber fracture is mainly induced by bend fracture failure. The machined surface is gradually smooth as the fiber orientation $\theta$ ranges from $80^{\circ}$ to $0^{\circ}$ or $\theta$ ranges from $150^{\circ}$ to $180^{\circ}$. The breaking point of the fiber is below the machined surface when the fiber orientation $\theta$ is between $80^{\circ}$ and $150^{\circ}$. The "cavities" machined surface can be easy to form. When $150^{\circ}<\theta \leq 180^{\circ}$, the fiber fracture is mainly induced by contact fracture failure. The machined surface is also smooth. 


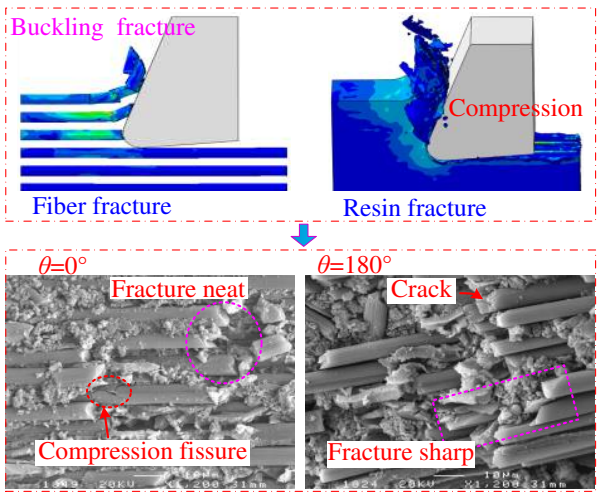

(a) Machined surfaces when $\theta=0 \% 180^{\circ}$

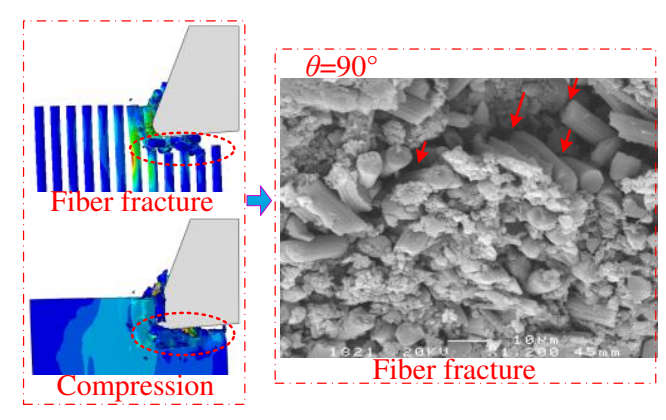

(c) Machined surfaces when $\theta=90^{\circ}$

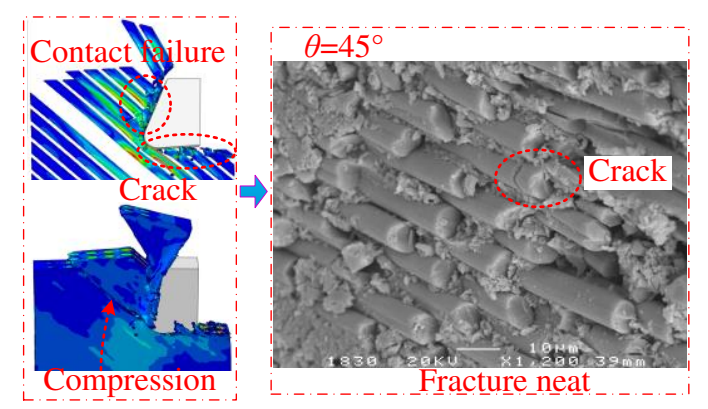

(b) Machined surfaces when $\theta=45^{\circ}$

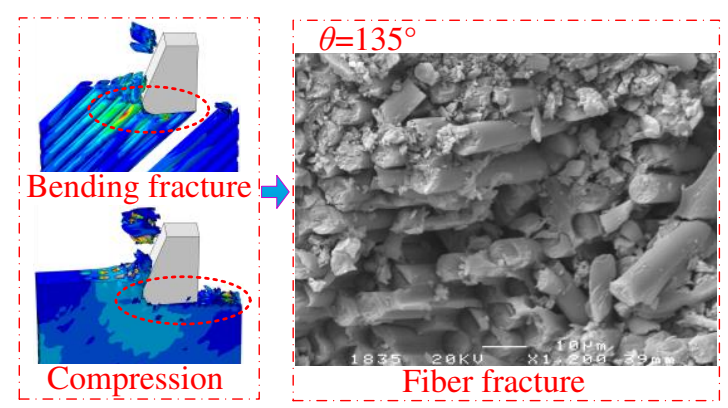

(d) Machined surfaces when $\theta=135^{\circ}$

Fig.9 Formation of machined surface under different cutting conditions

Additionally, when $80^{\circ}<\theta<180^{\circ}$, the fracture mode may be changed and the "cavities" machined surface may be formed. Thus, the damage depth of the fiber is investigated, as presented in Fig. 10. When $85.95^{\circ}<\theta<157.6^{\circ}$, with the increase of fiber orientation, the fiber damage depth increases first and then decreases. The damage depth reaches the maximum value of $42.3 \mu \mathrm{m}$ at $\theta=100.3^{\circ}$. The machined surface roughness changes are the greatest at this time. This is consistent with the conclusion obtained by $\mathrm{Hu}$ et al $[12,16,17-18]$. When $80^{\circ}<\theta<85.95^{\circ}$ and $157.6^{\circ}<\theta<180^{\circ}$, the fiber damage depth is less than $10 \mu \mathrm{m}$. Especially, when $169^{\circ}<\theta<180^{\circ}$, the fiber damage depth is less than $5 \mu \mathrm{m}$. According to the machined surface morphology of the groove wall, the machined surface is relatively smooth when the fiber orientation is near about $90^{\circ}$. With the increase of fiber orientation, the "cavities" machined surface gradually occurs. Then, the surface roughness is bound to increase. However, the 
machined surface appears the whole smooth again and the surface roughness is bound to decrease. Therefore, the results of cutting mechanics model and finite element model are basically consistent with the experimental observation. So, the cutting mechanics model and finite element model can effectively reflect the evolution law of CFRP machined surface.

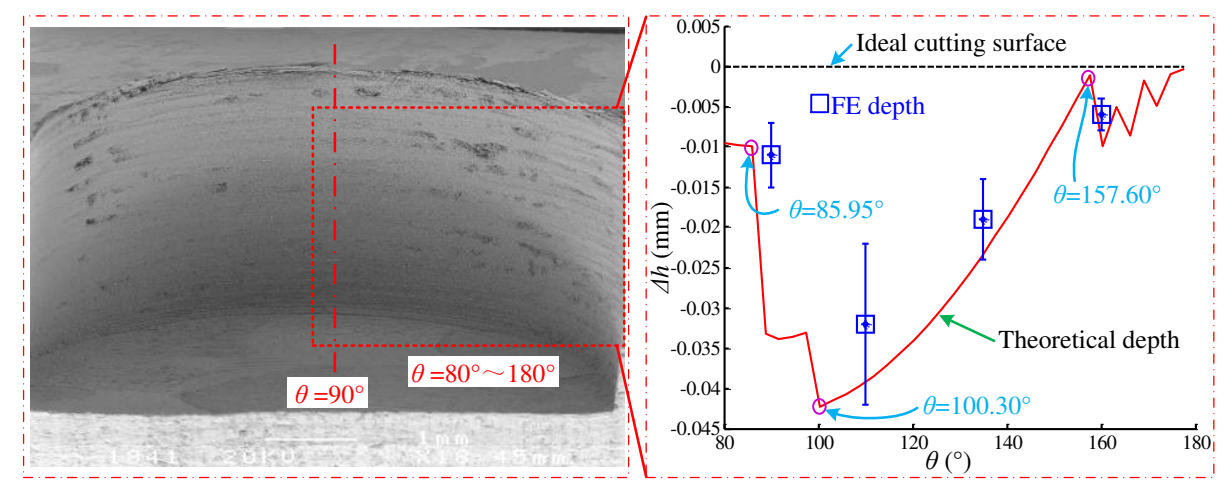

Fig.10 Variation of damage depth when $80^{\circ}<\theta<180^{\circ}$

\section{Conclusions}

In this paper, based on the elastic foundation beam theory and the Hertzian contact theory, the cutting mechanics are established. Meanwhile, the cutting model is simulated by the three-dimensional micro-scale numerical model. Then, the continuous varying cutting mechanism and the sub-damage are deeply studied in detail by combining the cutting mechanics model and the simulation model. Some key conclusions are drawn from the results presented in this research as follows:

(1) The fiber orientation $\theta=80^{\circ}$ and $\theta=150^{\circ}$ is the transition critical point of the fracture form. When $\theta=0^{\circ}$, the fiber failure is mainly buckling fracture. When $0^{\circ}<\theta<80^{\circ}$, the fiber failure is mainly contact fracture. When $80^{\circ}<\theta<150^{\circ}$, the fiber failure is mainly bending fracture. Then, the "cavity" machined surface may be formed because the fiber breaking point is under the cutter. When $150^{\circ}<\theta<180^{\circ}$, the fiber failure is mainly contact fracture.

(2) When the warp fiber direction $\theta$ is near $0^{\circ}, 45^{\circ}, 90^{\circ}$ and $180^{\circ}$, the machined surface of the 
milling groove wall is relatively smooth. Some "cavities" can be observed on the machined surface when $\theta$ is near $135^{\circ}$. When $\theta$ is near $45^{\circ}$, the machined surface is the smoothest. When $\theta$ is near $90^{\circ}$, the machined surface is smooth and clean.

(3) When $0^{\circ} \leq \theta \leq 80^{\circ}$, the fiber fracture is mainly caused by the contact fracture failure. The machined surface is generally smooth. When $80^{\circ}<\theta \leq 150^{\circ}$, the fiber fracture is mainly induced by bend fracture failure and the "cavities" machined surface can be easy to form. When $150^{\circ}<\theta \leq 180^{\circ}$, the fiber fracture is mainly induced by contact fracture failure. The machined surface is also smooth.

(4) When $85.95^{\circ}<\theta<157.6^{\circ}$, with the increase of fiber orientation, the fiber damage depth increases first and then decreases. The damage depth reaches the maximum value of $42.3 \mu \mathrm{m}$ at $\theta=100.3^{\circ}$. When $80^{\circ}<\theta<85.95^{\circ}$ and $157.6^{\circ}<\theta<180^{\circ}$, the fiber damage depth is less than $10 \mu \mathrm{m}$. Especially, when $169^{\circ}<\theta<180^{\circ}$, the fiber damage depth is less than $5 \mu \mathrm{m}$.

Author contribution Fei Su: Conceptualization, formal analysis, resources, writing-review and editing, supervision, project administration, funding acquisition. Chunjie Li: Methodology, validation, investigation, data curation, writing original draft. Guojun Dong: Modeling analysis, test operation, writing-review and editing (Sections2.1-2.2). Lei Zheng: Data curation, writing-review and editing (Section 2.3). Bing Chen: Test operation, Matlab programming.

Funding This work was financially supported by the National Science Foundation of China (No.52175400, No.51805164, No.52075127), Natural Science Foundation of Hunan Province (No. 2021JJ30263) and Natural Science Foundation of Jiangsu Province (No.BK20201474)

Data availability All data generated or analyzed during this study are included in this published article.

\section{Declarations}


Ethics approval Authors have read the "Ethical Responsibilities of Authors" and "Compliance with Ethical Standards." This study is an original paper which has neither previously nor simultaneously in whole or in part been submitted anywhere else. Results are presented clearly, honestly, and without fabrication, falsification, or inappropriate data manipulation. Complied fully with the COPE guidelines.

Consent to participate Not applicable.

Consent for publication Not applicable.

Competing interests The authors declare no competing interests.

\section{References}

1. Choudhury M R, Srinivas M S, Debnath K (2018) Experimental investigations on drilling of lignocellulosic fiber reinforced composite laminates. J Manuf Process 34:51-61.

2. Zhu Z, Guo K, Sun J, Li J, Liu Y, Zheng Y, Chen L (2018) Evaluation of novel tool geometries in dry drilling aluminium 2024-T351/titanium Ti6Al4V stack. J Mater Process Tech 259:270-281.

3. Wang X, Shen X, Zeng C, Sun F (2018) Combined influences of tool shape and as-deposited diamond film on cutting performance of drills for CFRP machining. Surf Coat Tech 347:390-397.

4. Loja M A R, Alves M S F, Bragança I M F, Rosa R S B, Barbosa I C J, Barbosa J I (2018) An assessment of thermally influenced and delamination-induced regions by composites drilling. Compos Struct 202:413-423.

5. Xu J, Li C, Mi S, An Q, Chen M (2018) Study of drilling-induced defects for CFRP composites using new criteria. Compos Struct 201:1076-1087.

6. Deng H, Xu Z (2019) Dressing methods of superabrasive grinding wheels: A review. J Manuf Process 45:46-69.

7. Wolfgang H, Felix B (2018) Influence of spatial tool inclination on delamination when milling CFRP. J Mater Process Tech 252:830-837.

8. Seyedbehzad G, Jean-Francois C, Gilbert L (2016) Finite element analysis of surface milling of carbon fiber-reinforced composites. Int J Adv Manuf Technol 87:399-409. 
9. Mei J W, Oriol G D, Dragos A A (2019) Modelling the unidirectional fiber composite milling force oscillations through capturing the influence of the stochastic fiber distributions. Compos Struct 226:1-15.

10. Noritaka K (2019) Evalution of internal defects generated in machine milled carbon fiber reinforced plastic using X-ray computed tomography. Precis Eng 60:257-264.

11. Wang F J, Zhang B Y, Zhao M, Cheng D, Wang Z G (2019) Evolution laws of fiber-matrix interface cracks in machining of carbon fiber reinforced polymer. Int J Adv Manuf Technol 101:963-977.

12. Hu N S, Zhang L C (2004) Some observations in grinding unidirectional carbon fiber-reinforced plastics. J Mater Process Tech 152:333-338.

13. Wang C Y, Liu G Y, An Q L, Chen M (2017) Occurrence and formation mechanism of surface cavity defects during orthogonal milling of CFRP laminates. Compos part B 109:10-22.

14. Niu B, Su Y L, Yang R, Jia Z Y (2016) Micro-macro-mechanical model and material removal mechanism of machining carbon fiber reinforced polymer. Int J Mach Tools Manuf 111:43-54.

15. Lukas S, Daniel S, Konrad W (2020) Analytical force model for drilling out unidirectional carbon fiber reinforced polymers (CFRP). J Mater Process Tech 278:1-23

16. Robert V, Lukas S, Friedrich K,Konrad W (2019) Analytical force model for orthogonal machining of unidirectional carbon fiber reinforced polymers (CFRP) as a function of the fiber orientation. J Mater Process Tech 263:440-469.

17. Alessandro A, Khamis E (2019) 3D micro-mechanical modelling of orthogonal cutting of UD-CFRP using smoothed particle hydrodynamics and finite element methods. Compos Struct 218:174-193

18. Alessandro A, Sein L S, Khamis E (2017) Modelling the orthogonal cutting of UN-CFRP composites: Development of a novel cohesive zone model. Compos Struct 168:65-83.

19. Su Y L (2019) Effect of the cutting speed on the cutting mechanism in machining CFRP. Compos Struct 220:662-676

20. Meng Q X, Cai J Cheng H, Zhang K F (2020) Investigation of CFRP cutting mechanism variation and the induced effects on cutting response and damage distribution. Int $\mathrm{J}$ Adv Manuf Technol 106:2893-2907.

21. W. Xu, L. C. Zhang (2014) On the mechanics and material removal mechanisms of vibration-assisted cutting of unidirectional fibre-reinforced polymer composites. Int $\mathbf{J}$ Mach Tools 
Manuf 80-81(5) : 1-10.

22. K L Johnson (1985) Contact Mechanics, Cambridge University press.

23. X Q Yang, S R Liu, Y Chu, S Qian (2015) Calculation on contact stress and plastic yield stability in bearings. Bearing 3: 7-10.

24. M Biot (1937) Bending of an infinite beam on an elastic foundation. J Appl Mech. 